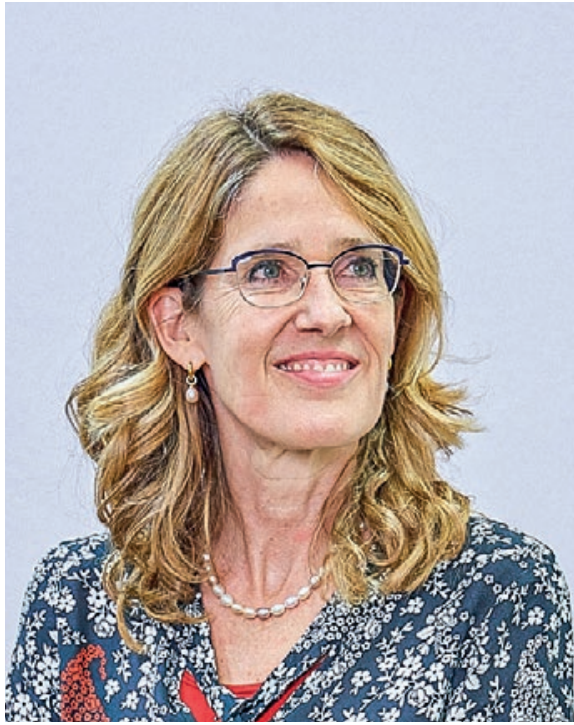

Dre méd. Rachel Enz Perschel

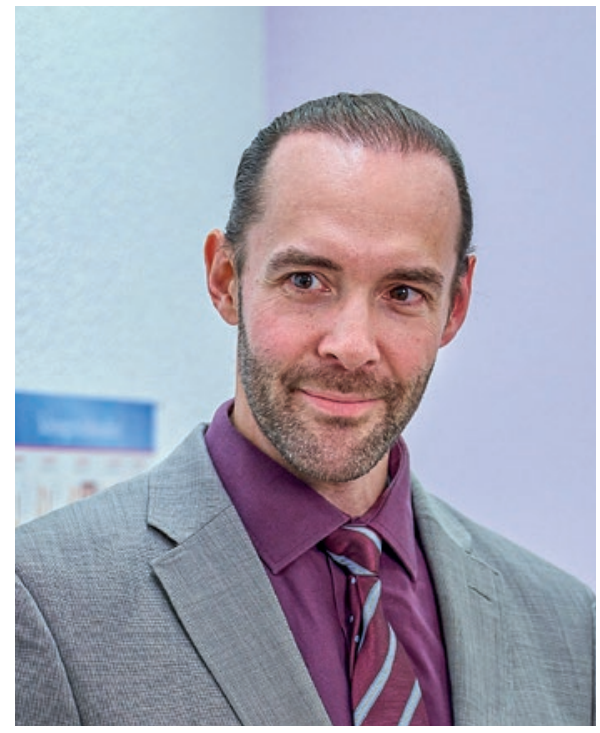

Dr iur. Kaspar Gerber

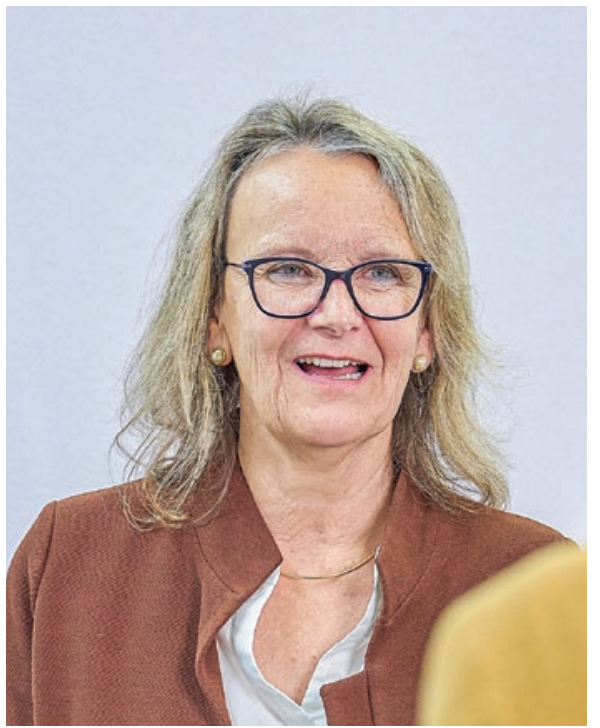

Dre théol. Ruth Baumann-Hölzle

\title{
La vaccination prise en tenaille
}

\section{Interview: Charlotte Schweizer}

Cheffe de la communication de la FMH

Le mécontentement monte vis-à-vis des mesures prises contre le coronavirus. Des taux de vaccination élevés font espérer une suppression des restrictions. On parle de plus en plus d'une vaccination axée sur certaines institutions ou certains groupes professionnels. Cela soulève de nombreuses questions. Dans notre interview, une médecin, une experte en éthique médicale et un juriste présentent leurs avis personnels. La FMH veut tenir compte de la complexité de ce thème et inviter à la réflexion et au dialogue nuancé.

Dans une pandémie, y a-t-il quelque chose comme une obligation morale de vaccination, Madame Baumann-Hölzle? Peut-on forcer les gens à se faire vacciner?

R. Baumann-Hölzle: En droit, toute forme d'acte médical remplit les conditions de l'état de fait qui caractérise une atteinte corporelle. En éthique, son pendant est l'atteinte à l'intégrité. Il est parfois envisageable qu'une obligation s'impose pour certains groupes de personnes, voire pour l'ensemble de la population, sous l'angle du principe de proportionnalité. Mais jusqu'à ce que l'Etat, garant de la démocratie, puisse ordonner une obligation vaccinale, il faut soupeser avec grand soin le droit de l'individu à son intégrité physique, psychique et sociale, l'introduction d'une contrainte vaccinale, et le droit à la protection contre la mise en danger de la santé de tiers. En dernière ana-

\section{Ont participé à cette interview:}

Dre Rachel Enz Perschel, médecin spécialiste en médecine interne générale, responsable des questions médicales du cabinet médical wollimed ag

Ruth Baumann-Hölzle, Dre en théologie, experte en éthique dans le domaine de la santé, responsable de la Fondation Dialogue Ethique

Kaspar Gerber, Dr en droit, LL.M, collaborateur scientifique (postdoc), chaire de droit constitutionnel, droit administratif et droit des assurances sociales, Université de Zurich

\section{L'interview a eu lieu le 10 novembre 2021.}

lyse, le droit à la protection est justifié par ce droit à de multiples intégrités. Dans une telle situation, les acteurs étatiques se trouvent dans un dilemme éthique. Quelle que soit leur décision, ils enfreindront des 
droits constitutionnels fondamentaux. Sur le plan des principes, en cas d'atteinte à l'intégrité, la question se pose de savoir quelle est son ampleur et s'il existe des solutions alternatives à plus bas seuil. Ainsi, l'obligation de test empiète de manière nettement moins forte que la vaccination, raison pour laquelle les possibilités de tests doivent d'abord être épuisées. Ce n'est que lorsqu'on a prouvé que les tests n'empêchent pas de manière comparable la contagion que l'introduction d'une obligation vaccinale peut être éthiquement défendable.

\section{Comment se présente la situation du point de vue juridique? L'Etat est-il autorisé à contraindre les gens à se faire vacciner, Monsieur Gerber?}

K. Gerber: Dans certaines conditions, une telle obligation est juridiquement admissible et défendable, c'est ce que dit la Loi sur les épidémies. Elle cite comme conditions préalables la proportionnalité et la subsidiarité. Une mesure est proportionnée lorsqu'elle est appropriée, nécessaire et raisonnablement exigible. Dans la situation particulière actuelle, le Conseil fédéral peut ordonner cette obligation pour des groupes de personnes menacés, pour des groupes de personnes particulièrement exposés, et pour des personnes qui exercent certaines activités. L'ordonnance explique qu'il ne faudrait recourir que de manière très ciblée à une obligation vaccinale, donc p. ex. pour le personnel de santé, uniquement dans les domaines où p. ex. le

\section{Dre méd. Rachel Enz Perschel: "Avec des argu- ments médicaux, on ne touche plus guère les gens qui se sont forgés une opinion.»}

risque de nouvelle propagation de la maladie est accru. Mais ici aussi, la subsidiarité s'applique, donc se faire tester plutôt que se faire vacciner. Car bien entendu, on ne peut contraindre physiquement personne à se faire vacciner.

\section{Comment la vaccination contre le Covid a-t-elle été perçue par vos patientes et vos patients, Madame Enz Perschel?}

R. Enz Perschel: Dans ma perception, près de $80 \%$ de nos patientes et de nos patients âgés de plus de 50 ans sont vaccinés. Je constate rarement un refus total de la vaccination. Au début, nous avons presque été submergés par les volontaires, et à l'heure actuelle, il arrive parfois que des personnes réclament leur dose avec insistance. Quelques femmes enceintes viennent au $4^{\mathrm{e}}$ mois pour se faire vacciner. Chez les plus de $80 \mathrm{ans}$, il $\mathrm{y}$ a le groupe de personnes en bonne santé et en bonne forme qui veulent absolument se faire vacciner. Il y a en outre des personnes âgées qui sont fatiguées, qui disent avoir vécu assez longtemps et qui s'accommodent désormais d'une contagion. Parmi les plus de

Dr iur. Kaspar Gerber: «Une telle obligation peut provoquer des réactions de rejet ou des pertes de personnel.»

90 ans, certains refusent même consciemment la vaccination. D'autres personnes qui ne veulent pas se faire vacciner se considèrent tout simplement comme non menacées. Elles justifient cela par le fait qu'elles habitent chez elles, qu'elles ne rencontrent que peu de gens, ou qu'elles pensent avoir de bons anticorps qui les mettraient à l'abri de la maladie.

\section{Du point de vue épidémiologique, des mesures} strictes devraient endiguer la pandémie le plus vite possible; du point de vue individuel, il ne faudrait pas toucher à la liberté individuelle et à l'autonomie. Comment évaluez-vous ce conflit d'objectifs?

K. Gerber: De manière tout à fait générale, il faut analyser continuellement la relation coût-bénéfice de différentes mesures de lutte contre la pandémie et soupeser leurs avantages et leurs inconvénients. Une obligation de vaccination, et aussi le certificat, exercent une pression en raison des inconvénients qui en résultent pour les non-vaccinés, p. ex. parce qu'on leur refuse l'accès à certains lieux publics comme les restaurants, les centres de fitness ou les maisons de re traite. Si le contrat de travail les oblige à se faire vacciner, il se peut que leur activité professionnelle soit menacée au cas où l'employeur n'autorise pas les tests. Outre l'aspect épidémiologique, il faut tenir compte du fait qu'une telle obligation peut provoquer des réactions de rejet, un contournement des mesures ou des pertes de personnel. A mes yeux, certaines mesures, telles que des restrictions d'accessibilité à des institutions publiques, sont appliquées aujourd'hui trop massivement au lieu d'être axées de manière plus spécifique sur les groupes à risque et sur des lieux pertinents du point de vue épidémiologique.

R. Baumann-Hölzle: En éthique, on distingue entre les activités du quotidien et les activités qui permettent de bien vivre. On considère que le fait d'empiéter sur la vie quotidienne, p. ex. ne plus être autorisé à faire ses achats ou à aller à l'école, est une atteinte massive à l'intégrité sociale; en revanche, des empiètements sur notre manière de bien vivre, comme $p$. ex. aller au restaurant, sont considérés comme moins pénalisants. Néanmoins, la question se pose de savoir dans quelle mesure on ne met pas ainsi en jeu la paix sociale, car il ne s'agit pas que de santé, mais de nombreuses autres 


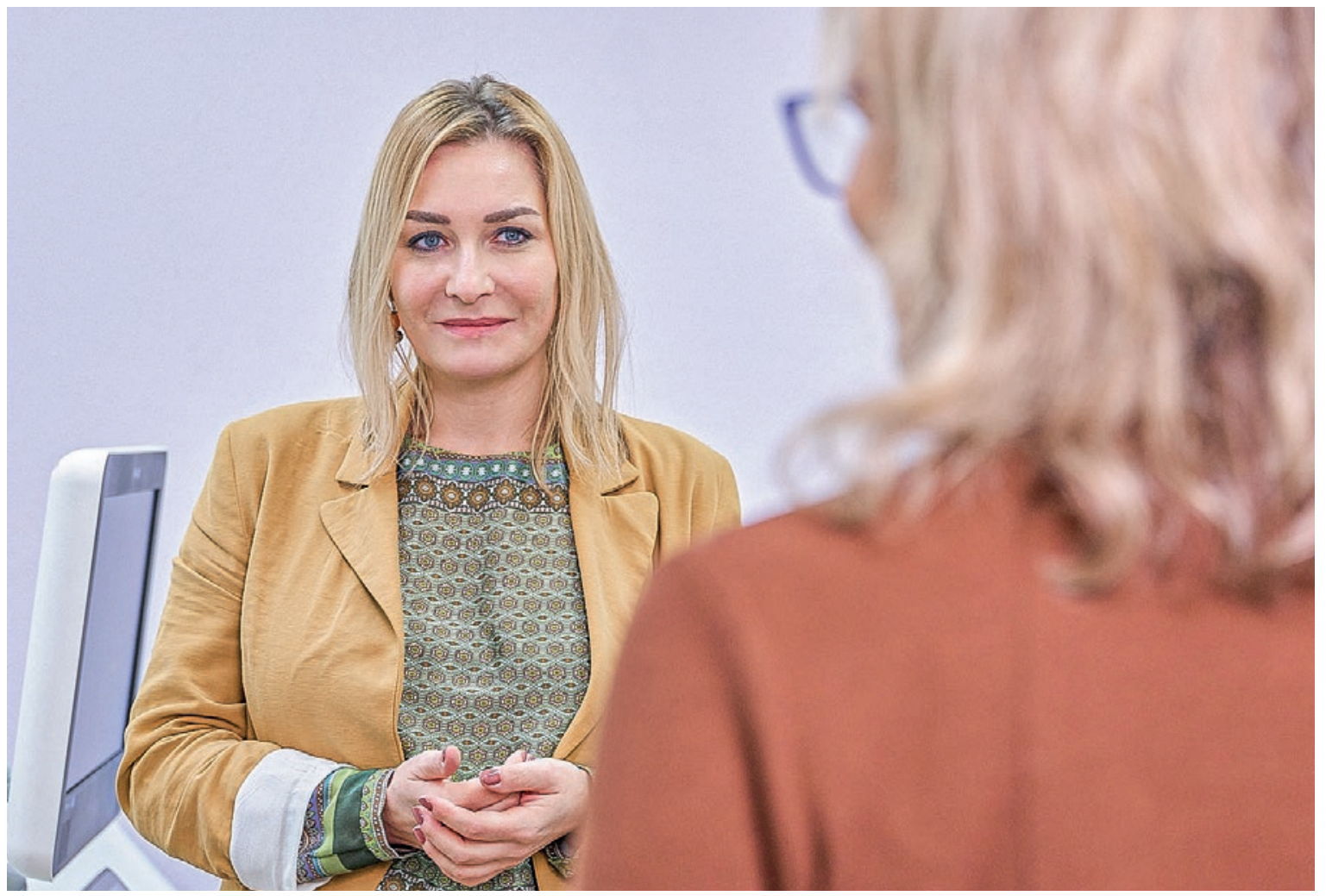

Charlotte Schweizer en conversation avec Ruth Baumann-Hölzle.

valeurs comme p. ex. la cohésion de la société, ou le droit à la liberté de mouvement. Il est donc très important de ne pas parler d'une obligation générale de vaccination, mais de considérer le risque de contagion en relation avec d'autres risques, et de différencier les mesures en fonction des différents groupes à risque. J'estime que cette réflexion fait actuellement défaut dans le discours public.

R. Enz Perschel: Plus les gens seront nombreux à être vaccinés, et plus vite on mettra un terme à la pandémie. Se faire tester ne la réduit pas. Les gens préféreraient ne pas devoir se faire vacciner et ne pas porter de masque. Mais en ce moment, il n'y a aucune alternative. Les informations et les recommandations sont le seul moyen de convaincre nos semblables de se faire vacciner. Mais j'ai l'impression qu'avec des arguments médicaux, on ne touche plus guère les gens qui se sont forgés une opinion.

Les enfants en bas âge ont un risque moindre de subir des atteintes durables en raison d'une infection au Covid. Quand on les vaccine, cela se fait en priorité pour protéger d'autres gens menacés par une contagion. Qu'en dites-vous?

R. Enz Perschel: A l'heure actuelle, nous ne savons pas encore quels sont les effets du vaccin sur les enfants. Je conseille aux parents de se faire vacciner afin de faire en sorte que la majorité d'entre eux soient protégés contre une éventuelle contagion due à leurs enfants, et d'éviter de devoir eux-mêmes se mettre en quarantaine. Ainsi, les enfants n'auront pas besoin d'avoir peur de contaminer leurs parents ou leurs grandsparents. Je peux partiellement comprendre l'organisation de parents Protect the Kids qui exige de faire vacciner les moins de 12 ans, surtout s'il s'agit d'enfants à haut risque présentant des affections cardiaques ou pulmonaires et qui sont de toute façon aussi vaccinés contre la grippe. Pour ce groupe d'enfants, on peut envisager une vaccination Covid en régime "off-label use», autrement dit en dérogation aux indications de l'étiquette (dans la situation actuelle, à savoir en novembre 2021). Dans d'autres pays, le vaccin est déjà autorisé pour les enfants de 5 à 11 ans.

K. Gerber: En ce moment, je ne vois pas les avantages médicaux des vaccins contre le Covid-19 pour les enfants, sauf pour certaines pathologies préexistantes. Il est délicat de leur dire en bloc: vous êtes tous des "propagateurs de virus», raison pour laquelle nous vous vaccinons - même si le risque pour vous-mêmes n'est que minime.

R. Baumann-Hölzle: Ce serait une instrumentalisation des enfants pour les parents non vaccinés ou pour les 
membres de groupes à risque qui ne veulent pas se faire vacciner. Cela ne doit pas se faire: les groupes à risque avaient la possibilité de se faire vacciner. Autre point problématique: comment un enfant qui n'a pas encore de projet de vie peut-il véritablement prendre une décision éclairée en ce qui concerne la vaccination, le cas échéant contre la volonté de ses parents? Cette question peut semer la zizanie entre parents et enfants.

\section{Quelles sont, à votre avis, les raisons du scepticisme vis-à-vis de la vaccination?}

R. Enz Perschel: Surtout au début de la pandémie, le fait d'avoir été souvent mal informés, voire d'avoir été souvent informés de manière erronée par les autorités, n’a pas aidé. Je me souviens du message de l'OFSP, révisé par la suite, selon lequel "Les masques sont inutiles», qui avait été suivi par un manque d'approvisionnement en masques et autre matériel de protection. Ainsi, des groupements qui ne croient plus en rien se sont formés. Autres raisons possibles: la peur et le manque de connaissances. De nombreux jeunes redoutent une inflammation du muscle cardiaque qui peut être déclenchée par la vaccination mais sans voir qu'il est plus fréquent qu'une inflammation du muscle cardiaque soit due à une infection au coronavirus qu'à la vaccination. Ou le mensonge sur l'infertilité qui serait soi-disant due au Covid, un mensonge que même une déclaration vidéo scientifiquement fondée publiée sur le site de l'OFSP ne peut apparemment pas réfuter.

R. Baumann-Hölzle: Vous me faites penser aux retards de règles que de nombreuses femmes ont vécu après la vaccination, et qui n'ont souvent pas été pris au sé-

Dre théol. Ruth Baumann-Hölzle: "Je me demande si nous arriverons à réactiver les principes de l'éthique médicale après la pandémie.»

rieux du côté des médecins, et aussi des autorités. On pourrait en déduire que si de tels incidents ne sont pas pris au sérieux, peut-être que cette histoire d'infertilité contiendrait quand même quelques lueurs de vérité? Je veux dire par là que certaines erreurs de communication et de contenu se sont produites qui ont durablement troublé la confiance de nombreuses personnes dans les autorités. Le Conseil fédéral a par exemple déclaré à plusieurs reprises que lorsque tous ceux qui souhaitent se faire vacciner et que tous les patients à risque auront été vaccinés, on suspendra les mesures. Et maintenant que c'est le cas, on nous dit que nous devons quand même les conserver, car sinon, les services de soins intensifs seront surchargés.
K. Gerber: Il va de soi que la vaccination est, à l'heure actuelle, une mesure de prévention très efficace pour certains groupes de personnes et qu'elle déploie ses effets en quelques semaines seulement. Toutefois, on a proclamé que la vaccination est un moyen de changer radicalement la donne de la pandémie, bien qu'on ait vite appris, à l'échelle internationale également, que cette déclaration ne saurait être valable de manière aussi absolue. Il est donc compréhensible que certaines personnes soient frustrées - qu'il s'agisse de personnes vaccinées ou non vaccinées. Le fait que le message de la campagne de vaccination soit «Tu dois protéger les

Dre méd. Rachel Enz Perschel: «Selon l'état actuel de nos connaissances, les personnes vaccinées sont moins infectieuses."

autres» au lieu de "Protège-toi toi-même!» me semble être un problème fondamental. Cela exerce une pression à se faire vacciner au nom de la solidarité, ce qui suscite des aversions et des contre-pressions. A mes yeux, cela n'a rien à voir avec un égoïsme nuisible à la société, c'est une mesure d'autodéfense qui correspond tout simplement à l'objectif principal de la vaccination.

\section{Frank Ulrich Montgomery, le président du comité de l'Association médicale mondiale, parle d'une "tyran- nie des non-vaccinés exercée sur les deux tiers des vaccinés» qui imposent les mesures à tous les autres. Comment jugez-vous cette déclaration?}

R. Enz Perschel: Selon l'état actuel de nos connaissances, les personnes vaccinées sont moins infectieuses lorsqu'elles attrapent le virus. La vaccination n'est toutefois pas une protection à cent pour cent. Dans cette mesure, même les vaccinés ne peuvent échapper à l'obligation générale de porter un masque et de respecter les distances entre les personnes.

K. Gerber: Compte tenu du fait que le nombre de cas augmente malgré la vaccination et que d'autres pays, qui ont parfois des taux de vaccination très élevés, se mettent petit à petit à resserrer la vis, la déclaration de Monsieur Montgomery est problématique. Il collectivise la responsabilité pour la santé, donc la protection des autres. La vaccination devient quasiment l'objet d'une déclaration de nature politique et sociétale. Etre vacciné devient un label de qualité que les gens affichent parfois fièrement, en montrant leurs deux piqûres sur les réseaux sociaux. La polarisation s'accroît: vacciné ou non vacciné? Pour ou contre la Loi Covid et le Conseil fédéral? Le statut vaccinal polarise.

R. Baumann-Hölzle: Dans ses critiques, Monsieur Montgomery devrait se focaliser avec des paroles moins agressives sur les groupes qui ne se font pas vac- 
ciner malgré un risque élevé, au lieu de qualifier de tyranniques tous les non-vaccinés sans distinction aucune. On en vient ainsi à condamner des gens sans connaître leurs motivations. Dans la mesure où tous ceux qui ne pensent pas comme tout le monde sont traités comme des adversaires, comme des ennemis, on est déjà face à une escalade. De telles généralisations à l'emporte-pièce sont très dangereuses, car elles mettent de l'huile sur le feu en montant certaines catégories de population les unes contre les autres.

\section{Il y a des institutions publiques qui donnent des autocollants en or ou en argent pour caractériser le statut «vacciné» ou "testé». Qu'en pensez-vous?}

K. Gerber: En fait, tant le statut de vacciné que celui de testé reste une affaire privée. Cette protection de la sphère privée a été déjà sapée massivement par le certificat.

R. Baumann-Hölzle: La thèse suivante se cache derrière les autocollants en or et en argent: avec la vaccination, nous maîtrisons toute la situation, ce qui n'est pas le cas. Il n'existe aucun droit de renforcer de cette manière la ségrégation entre vaccinés et non-vaccinés. Je constate que dans la pandémie, de nombreux principes d'éthique médicale ont été vidés de leur substance, c.à-d. qu'ils ne sont plus respectés comme ils devraient l'être. Je considère par exemple comme hautement douteuse la manière dont le secret médical a été grignoté, tout comme la façon dont les employeurs sont autorisés à consulter les données de santé de leur personnel. Et je me demande si nous arriverons à réactiver les principes de l'éthique médicale après la pandémie.

\section{Quels sont pour vous les plus grands défis pour notre} société lorsqu'il s'agit d'enrayer la pandémie?

K. Gerber: Pour ce qui est des virus respiratoires, il s'agit en principe d'un risque de vie courant et accentué par l'effet saisonnier. Dès lors, des contre-mesures appliquées de manière généralisée comme la distanciation sociale, en fonction de leur degré d'intensité, peuvent provoquer immédiatement des dommages collatéraux sur l'ensemble de la société. A l'heure actuelle, la gestion des risques de pandémie par l'Etat sollicite de très nombreuses ressources; à un moment donné, on atteindra le point où, pour cette raison, on aura trop longtemps négligé d'autres risques de vie ainsi que les personnes touchées par ces risques, de sorte qu'il deviendra difficile de généraliser des me- sures et, par conséquent, de respecter l'égalité de droit. Nous devrions peu à peu passer à une stratégie qui vise en priorité à informer sur le coronavirus en tant que risque de vie et, le cas échéant, émettre à l'avenir également des recommandations différenciées selon les groupes concernés.

R. Enz Perschel: Les jeunes souffrent particulièrement des mesures anti-Covid et en subiront encore longtemps les conséquences. A l'heure actuelle, nous constatons une recrudescence des problèmes psychiques, surtout chez les jeunes.

R. Baumann-Hölzle: La justice intergénérationnnelle est l'un des nombreux problèmes d'équité que nous connaissons déjà depuis longtemps, mais qui remonte maintenant à la surface sous l'effet de la pandémie. Il est certain qu'une société qui tolère des problèmes d'inégalité à un tel degré - l'inégalité de traitement entre les personnes affectées par la maladie, la charge exercée par les conditions de travail, particulièrement

Dre méd. Rachel Enz Perschel: «Nous constatons une recrudescence des problèmes psychiques, surtout chez les jeunes.»

dans le secteur de la santé - tout cela est un terrain fertile qui nourrit les agressivités. A certains endroits, cela a déjà engendré un "stress» moral, des blessures morales, voire des effondrements: on ne peut plus se mettre à la place des autres, on ne s'écoute plus, on reste inaccessible aux arguments basés sur les faits. C'est sur ces points qu'en tant que société, nous devons travailler d'urgence. Et il est tout à fait certain que l'Etat devra essayer de regagner la confiance des groupes de personnes qu'il a ébranlée, notamment en raison de ses promesses non tenues et de son manque de transparence.

Cet entretien est la troisième et dernière partie de la brève série d'articles sur l'obligation vaccinale. En $1^{\text {ère }}$ partie, la Dre iur. Iris Herzog-Zwitter a abordé l'«Obligation de vaccination: le cadre juridique» (Bull Med. 2021;102(47):1556-9). Le Dr iur. Kaspar Gerber s'est penché dans la $2^{e}$ partie sur le «Covid-19: proportionnalité des incitations à la vaccination" (Bull Med. 2021;102(48):1599-1601).

\section{Crédits photos}

Christian Jäggi 